\title{
Making Concentrated Pterostilbene Highly Bioavailable in Pressure Processed Phospholipid Nanoemulsion
}

\author{
Fu-Min Sun ${ }^{1}$, Yu-Jou Chou ${ }^{1}{ }^{1}$, Qingrong Huang ${ }^{2}$, Jing-Yu Hu ${ }^{1}$ and Yuwen Ting ${ }^{1, *(1)}$ \\ 1 Graduate Institute of Food Science and Technology, National Taiwan University, No. 1, Sec. 4, Roosevelt Rd., \\ Taipei City 106319, Taiwan; r06641030@ntu.edu.tw (F.-M.S.); f06641022@ntu.edu.tw (Y.-J.C.); \\ jingyu.huu@gmail.com (J.-Y.H.) \\ 2 Food Science Department, Rutgers University, 65 Dudley Rd., New Brunswick, NJ 08901-8525, USA; \\ qhuang@aesop.rutgers.edu \\ * Correspondence: pywting@ntu.edu.tw; Tel.: +886-2-3366-1878
}

Citation: Sun, F.-M.; Chou, Y.-J.; Huang, Q.; Hu, J.-Y.; Ting, Y. Making Concentrated Pterostilbene Highly Bioavailable in Pressure Processed Phospholipid Nanoemulsion. Processes 2021, 9, 294. https:// doi.org/10.3390/pr9020294

Academic Editor: Jer-An Lin

Received: 14 January 2021

Accepted: 25 January 2021

Published: 3 February 2021

Publisher's Note: MDPI stays neutral with regard to jurisdictional claims in published maps and institutional affiliations.

Copyright: (c) 2021 by the authors. Licensee MDPI, Basel, Switzerland. This article is an open access article distributed under the terms and conditions of the Creative Commons Attribution (CC BY) license (https:// creativecommons.org/licenses/by/ $4.0 /)$.

\begin{abstract}
Pterostilbene, a dimethylether analog of resveratrol, has been found to have potent biological activity. However, the bioavailability of pterostilbene in the biological system is limited due to its poor solubility in an aqueous environment. A nanoemulsion system was designed for this purpose. Lecithin-based nanoemulsion was formed after 3 cycles through a high-pressure homogenizer at 500 psi. The rheological properties and particle size were measured using dynamic light scattering and a viscometer. The storage stabilities of the prepared formulation were determined based on its ability to maintain its particle size and loading concentration. According to the experimental results, the lecithin-based nanoemulsion system contained approximately $9.5 \%$ of pterostilbene. Over the 28-day stability test, the particle size, zeta potential, and encapsulation of pterostilbene in the nanoemulsion did not change significantly, indicating good storage stability. The positive effect of the prepared nanoemulsion system on bioavailability was studied and confirmed using in vitro lipolysis and a caco-2 monolayer model.
\end{abstract}

Keywords: pterostilbene; nanoemulsion; lecithin; bioavailability; encapsulation

\section{Introduction}

Pterostilbene, trans-3,5-dimethoxy-4-hydroxystilbene, is found in many fruits and vegetables including blueberries, grapes, and tree woods [1-3]. Pterostilbene has attracted great attention from researchers due to its strong antioxidant potential and broad range of biological activities, such as anti-tumorigenesis, anti-inflammation, and prevention of the metabolic syndromes [3,4]. Furthermore, many researchers even found pterostilbene a more potent inhibitor to the development of chronic diseases, such as neurological diseases, chronic inflammation, and the growth of tumor, than its most well-studied analog, resveratrol [4-7]. Though pterostilbene has great potential in disease prevention, its poor aqueous solubility and stability seriously limit its application in the pharmaceutical and food industries.

The oral bioavailability of a bioactive compound is mainly affected by three factors: the accessibility to the absorption site, the transport coefficient across the intestinal lining, and its ability to escape from extensive metabolic activities in the living system [8]. Specifically, the bioaccessibility of orally ingested components is greatly affected by their solubility and stability in the aqueous intestinal lumen. For an unstable hydrophobic compound like pterostilbene, it has a very limited chance to come into contact with the enterocytes and, thus, needless to say, to get across the intestinal lining. Hence, it is critical to find a suitable delivery system that could protect and improve the solubility of pterostilbene.

Nanoemulsion is a thermodynamically unstable emulsion system, which is composed of two immiscible liquids mixed along with an emulsifying agent [9]. The general definition of a nanoemulsion is an emulsion system that has a droplet size beneath $500 \mathrm{~nm}$, more 
specifically $200 \mathrm{~nm}$ [10]. The smaller droplet size of nanoemulsion allows higher kinetic stability and intestinal permeability. When being used as the delivery vehicle for bioactive components, nanoemulsion is known for its high versatility to be tailored for encapsulation of various compounds and broad applicability in pharmaceutical or food matrixes.

The selection of emulsifier is one of the critical factors that significantly influences the stability of such a system, which is heavily influenced by the ability of its surfactant to provide suitable hydration, electrostatic repulsion, and steric stabilization [11-13]. Having two nonpolar fatty acid tails and a polar phosphate-choline head, lecithin was reported to contribute good hydration property to the emulsion system as well as being employed as a steric and electrostatic stabilizer [14]. In conjunction with the fact that it is natural, fully biocompatible, and generally recognized as safe, lecithin, with no doubt, has become a popular emulsifier to stabilize the colloidal system in recent years [15]. Successful cases of using lecithin for the development of nanoemulsion have been described in numerous preceding publications [16-18].

In the present study, high loading of pterostilbene was encapsulated in a lecithinbased nanoemulsion system. The optimum formulation and processing parameter was systematically studied and evaluated. The effects of the nanoemulsion system on the stability, antioxidant activity, and bioavailability of pterostilbene were examined using in vitro chemical and cell line essays. Overall, the nanoemulsion system developed in this work by far contains the highest amount of pterostilbene among the available literature. This exceptional stability and ability to preserve the bioactivity of pterostilbene also made the present nanoemulsion a successful demonstration of using such a system to improve the biological efficiency of hydrophobic compounds.

\section{Materials and Methods}

\subsection{Materials}

Pterostilbene (trans-3,5-dimethoxy-4-hydroxystilbene, purity >98\%) was bought from J\&K company (Beijing, China). Materials used to prepare nanoemulsion including medium chain triglyceride and liquid soybean lecithin were purchased from Bio-unique Medisupple (New Taipei city, Taiwan) and Confong Corp. (Taipei, Taiwan). Double deionized water was produced using a Milli-Q-water purification system (Millipore, MA, USA). For LC/MS/MS study, all chemicals were bought from Merck (Darmstadt, Germany). Ascorbic acid, dimethyl sulfoxide (DMSO), sodium taurodeoxycholate hydrate, sodium chloride, TRIS maleate salt, and 70-dichlorofluorescein diacetate (DCFH-DA) were bought from Sigma Aldrich Chemical Co. (St. Louis, MO, USA). Dulbecco's modified Eagle medium (DMEM), Hanks' balanced salt solution (HBSS), and penicillin/streptomycin solution were purchased from Thermo Fisher Scientific (Grand Island, NY, USA). Fetal bovine serum (FBS) was obtained from Biological Industries (Kibbutz Beit Haemek, Israel).

\subsection{Solubility Study}

To determine the carrier oil for the emulsion system, the solubility of pterostilbene in 7 different oils including peanut oil, palm oil, linseed oil, medium chain triglycerol, coconut oil, corn oil, and olive oil was evaluated. To start the solubility test, $1 \mathrm{~mL}$ of experimental oil containing $25 \mathrm{mg}$ of pterostilbene was heated and maintained at $60^{\circ} \mathrm{C}$ with continuous stirring on a heating magnetic stir plate. Once the compound was completely solubilized, $25 \mathrm{mg}$ of pterostilbene was added repetitively until the added compound could no longer be solubilized (turbidity prolongs for $1 \mathrm{~h}$ ). Later, the oil mixture was stored at room temperature $24 \mathrm{~h}$ before being subjected to filtration using a $0.45 \mu \mathrm{m}$ filter. The concentration of pterostilbene in the oil was then determined using a HPLC system.

\subsection{Determination of Emulsion Formulation Using the Pseudo-Ternary Phase Diagram}

The pseudo-ternary phased diagram was constructed by mixing the oil, emulsifier, and water at desired ratios using a method reported in a publication by Donsì et al. [19] with slight modification. The selected emulsifier (liquid lecithin) was first mixed with the oil 
phase at levels of $2.5 \%, 5 \%, 7.5 \%$, and $10 \%$. The prepared oil and emulsifier mixtures were then combined with DI water (Deionized water) using the aqueous titration method, by which the ratio of DI water was gradually increased. After being subjected to a high-speed homogenizer (T25 digital, IKA, Königswinter, Germany) running at 25,000 rpm for two minutes, samples were left standing in the dark for one hour at room temperature. After standing time, the physical status of the emulsion mixtures was observed and recorded. Based on the information obtained from the pseudo-ternary diagram, the formulations that will give a stable, homogeneous oil in water $(\mathrm{O} / \mathrm{W})$ emulsion could be easily found. Later, these formulations were subjected to series of physical studies for testing of their suitability for further high-pressure homogenization processing, which only allows the emulsions with viscosity less than $500 \mathrm{cps}$ and particle sizes smaller than $5 \mu \mathrm{m}$ to pass through.

\subsection{Preparation of Pterostilbene Nanoemulsion}

Combining the information obtained from the solubility study and pseudo-ternary phase diagram, the formulation used to prepare the pterostilbene nanoemulsion was decided. The oil that could solubilize the highest amount of pterostilbene was selected as the carrier oil to prepare the emulsion. To prepare the pterostilbene nanoemulsion, oil phase composed of carrier oil, emulsifier (soybean lecithin), and pterostilbene was first mixed at $35{ }^{\circ} \mathrm{C}$ with continuous stirring on a heating magnetic plate until a clear homogenous oily solution appeared, indicating the complete solubilization of all components. Afterward, the oil phase was mixed with the aqueous phase using a high-speed homogenizer (T25 digital, IKA, Germany) running at 25,000 rpm for 2 min to produce a coarse emulsion. Later, the nanoemulsion was formed by further processing the coarse emulsion with a high-pressure homogenizer (N-2 Nanolyzer, Gogene Co., Hsinchu, Taiwan) at a pressure level of 500 psi for 1-5 cycles. The prepared pterostilbene nanoemulsion emulsions were immediately subjected to physical and chemical evaluations.

\subsection{Particle Size and Zeta Potential Analysis}

The size and zeta potential of the droplet from the prepared pterostilbene nanoemulsions were analyzed using a dynamic light scattering spectrometer, 90Plus/BI-MAS (Brookhaven Instruments Corporation, Holtsville, NY, USA). The measurement was made using a solid-state laser operated at $660 \mathrm{~nm}$ at a fixed $90^{\circ}$ incidence angle under room temperature $\left(25^{\circ} \mathrm{C}\right)$. To prevent multiple scattering effects, droplets from various formulations and parameters were first diluted 10,000 times with DI water before being transferred to the $1-\mathrm{cm}$ path length cuvette for reading. The polydispersity indexes of the particles were obtained from the three consecutive measurements of each sample.

\subsection{Viscosity of Pterostilbene Nanoemulsion}

The viscosities of the emulsion system were measured using a Brookfield Viscometer (DVELV, Brookfield, Toronto, ON, Canada) at constant temperature $\left(25 \pm 0.1^{\circ} \mathrm{C}\right)$ at $100 \mathrm{rpm}$. Before sending to the high-pressure homogenizer, all of the coarse emulsion samples were subjected to viscosity testing since the available high-pressure homogenizer will only allow samples with a viscosity less than 500 centipoises to pass through without causing a serious instrumental blockage. Once the suitable formulation was decided, they were sent to go through 1-5 higher pressure homogenization cycles and the change of the viscosity value was again determined and recorded. The runs for the viscosity measurement were carried out $1 \mathrm{~h}$ and/or $24 \mathrm{~h}$ after the preparation.

\subsection{HPLC Analysis of Pterostilbene}

The concentration of pterostilbene was determined using a HPLC system (Shimadzu, Kyoto, Japan) consisting of a quaternary pump (L-7100), a degasser (ERC-3415 $\alpha$ ), and a variable wavelength UV/Vis detector (SPD-M20A). The analytical protocol was developed in reference to the method described by Liu et al. [20] with minor modifications. The HPLC system was operated using a binary gradient condition and pterostilbene was separated 
using a C18 reverse-phase column $(250 \times 4.6 \mathrm{~mm}, 5 \mu \mathrm{m})$ and detected at $306 \mathrm{~nm}$. The flow rate of mobile phase $\mathrm{A}$ (acetonitrile) and mobile phase $\mathrm{B}$ (double deionized water) were both set at $1 \mathrm{~mL} / \mathrm{min}$. The total running time of the HPLC analysis was $40 \mathrm{~min}$. Initially, the mobile phase was set at 30\% A and then increased linearly to $90 \%$ at $0-15$ min, held for $5 \mathrm{~min}$, then decreased to $30 \%$ within $1 \mathrm{~min}$, and again equilibrated for $14 \mathrm{~min}$. The sample injection volume was $10 \mu \mathrm{L}$ for all sample.

\subsection{Encapsulation Efficiency of Pterostilbene Nanoemulsion}

The encapsulation efficiency (EE) of pterostilbene in the nanoemulsions was determined using the ultrafiltration method described in literature published by Diane et al. [21] with slight modification. The centrifugal filter devices (Merck Millipore Amicon Ultra$0.5,50 \mathrm{kDa}$ MWCO) were used to separate the amount of pterostilbene in the external aqueous phase (the free compound) from those that were contained in the nanoemulsion droplets. The testing samples were subjected to $30 \mathrm{~min}$ of centrifugation (Hitachi CF15RX II, Tokyo, Japan) at $5500 \mathrm{rpm}$ at $4{ }^{\circ} \mathrm{C}$. The concentrated liquids were collected and diluted using methanol at 1 to 9 ratio. After dilution, the sample was filtered using a $0.45 \mu \mathrm{m}$ PVDF filter (Millipore Millex-HV) and then subjected to HPLC for the determination of the pterostilbene concentration.

\subsection{In Vitro Lipolysis of Nanoemulsion}

Lipolysis experiment is a convenient way to mimic the digestion activity and to evaluate the bioaccessibility of a formulation [22]. The in vitro lipolysis study was conducted using the protocol described in our previous published paper with slight modification [8]. In short, a fasted-state lipolysis buffer was prepared with $50 \mathrm{mM}$ of Tris maleate, $150 \mathrm{mM}$ of $\mathrm{NaCl}, 5 \mathrm{mM}$ of $\mathrm{CaCl}_{2} \mathrm{H}_{2} \mathrm{O}, 20 \mathrm{mM}$ of NaTDC, and $5 \mathrm{mM}$ of phosphatidylcholine. Freshly prepared pancreatin was made by mixing $1 \mathrm{~g}$ of pancreatin powder with $5 \mathrm{~mL}$ of lipolysis buffer and then centrifuged at $2000 \mathrm{rpm}$. To prepare the oil and water suspension, an equivalent concentration of pterostilbene as if in the emulsion formulation was added to either oil or double deionized water, respectively. For the lipolysis experiment, an emulsion and oil suspension containing 250mg of lipid was sampled while the water suspension was collected in the same volume as the oil suspension group. The pre-collected experimental samples were then added to $1 \mathrm{~mL}$ of prepared pancreatin solution and mixed with $9 \mathrm{~mL}$ of lipolysis buffer. During the 2-h experimental period, the temperature was kept constant at $37 \pm 1{ }^{\circ} \mathrm{C}$ and the $\mathrm{pH}$ was maintained at $7.50 \pm 0.02$ using $0.25 \mathrm{~N} \mathrm{NaOH}$ titration. The volume of $\mathrm{NaOH}$ added each time was recorded and plotted against the time. Upon of the experiment, all of the lipolysis solutions were ultracentrifuged (type 60 rotor, Beckman Coulter, Brea, CA, USA) for $1 \mathrm{~h}$ at 50,000 rpm. After ultracentrifugation, only the middle layer of supernatant was collected and stored at $-80^{\circ} \mathrm{C}$ for later HPLC analysis. For HPLC analysis, $100 \mu \mathrm{L}$ of lipolysis supernatant sample was filtered using $0.22 \mu \mathrm{m}$ filter before being mixed with $900 \mu \mathrm{L}$ of methanol.

\subsection{DPPH Antioxidant Analysis}

The antioxidant activity of pterostilbene was determined using the DPPH (2,2-diphenyl1-picrylhydrazyl) free radical scavenging method published previously [23]. To evaluate the effect of the carrier system on the antioxidant activity of pterostilbene, the DPPH scavenging ability of pterostilbene suspended in oil was also evaluated and compared with those encapsulated in the nanoemulsion system. Testing samples (in the form of oil suspension or nanoemulsion) were diluted to contain $0.5,1,2$, and $3 \mathrm{mg} / \mathrm{mL}$ of pterostilbene. Later, $30 \mu \mathrm{L}$ of diluted samples were mixed with $870 \mu \mathrm{L}$ of $0.004 \% w / v$ DPPH-ethanol solution and were allowed to react for $30 \mathrm{~min}$. Two hundred microliters of reacted samples were then transferred to a 96-well microplate and their absorbance at $515 \mathrm{~nm}$ was read using a Biotek Epoch ${ }^{\mathrm{TM}}$ Microplate Spectrophotometer (BioTek Instruments, Winooski, VT, USA). Then, DPPH scavenging activity (\%) was calculated using the following formula:

$$
\text { DPPH scavenging activity }(\%)=\left[\left(\mathrm{A}_{\text {control }}-\mathrm{A}_{\text {sample }}\right) / \mathrm{A}_{\text {control }}\right] \times 100
$$




\subsection{Stability Test}

It is desirable to have an emulsion system that could be stable for a longer period of time. Here, the stability and quality changes of emulsions produced using various processing parameters were evaluated over 28 days. The qualities of emulsion samples were examined by monitoring the changes of the droplet size, zeta potential, viscosity, and encapsulation efficiency over the experimental period at $25^{\circ} \mathrm{C}$. All emulsion samples used in this study were processed in one single batch and three replicates were made for each measurement.

\subsection{Cell Culture}

Caco- 2 cell was cultured in DMEM medium (Thermo, New York, NY, USA) supplemented with $10 \%$ of fetal bovine serum, $1 \%$ penicillin/streptomycin solution, and $1 \%$ non-essential amino acid in an incubator maintained at $37^{\circ} \mathrm{C}$ with $5 \% \mathrm{CO}_{2}$ and $95 \%$ air. All cells were used in the passages of 10 to 30 .

\subsection{Cell Viability Test}

Caco-2 cell was seeded in the 96-well plate at the density of $2 \times 104$ cells per well. After being incubated for $24 \mathrm{~h}$, growth medium was replaced by $200 \mu \mathrm{L}$ of DMEM medium containing various amounts of 25 to $200 \mu \mathrm{M}$ pterostilbene. After being treated with pterostilbene for $24 \mathrm{~h}$, the treatment media was again replaced by $100 \mu \mathrm{L}$ fresh media containing $2 \mathrm{mg} / \mathrm{mL}$ of 3-[4, 5-dimethylthiazol-2-yl]-2, 5 diphenyl tetrazolium bromide (MTT, Sigma-Aldrich, St. Louis, MO, USA). After $4 \mathrm{~h}$ of incubation, the MTT medium was carefully aspired and $100 \mu \mathrm{L}$ DMSO was added to each well. The absorbance at $570 \mathrm{~nm}$ was measured using a microplate reader (BioTek Instruments, Winooski, VT, USA) after being stabilized for $15 \mathrm{~min}$ in the dark. The results were presented as the percentages of cell viability in relation to the blank control group [24].

\subsection{Caco-2 Transport Assay}

The caco-2 transport assay was executed using the protocol published by Hubatsch et al. in 2007 with slight modification [25]. The caco-2 cell was diluted to the concentration of $5 \times 10^{5} \mathrm{cell} / \mathrm{mL}$ before being seeded to a polyethylene terephthalate transwell insert $\left(0.9 \mathrm{~cm}^{2}\right.$, Corning Costar Co., Corning, NY, USA) in a 12-well plate. DMEM medium at a volume of $0.5 \mathrm{~mL}$ and $1.5 \mathrm{~mL}$ was added to the apical and basolateral side, respectively. The culture medium was changed every other day for the first 7 days, after which the medium was changed every day for at least 14 days. After 21-28 days of cultivation on the transwell insert, the cultivating medium has carefully aspired and $0.5 \mathrm{~mL}$ HBSS buffer containing $50 \mu \mathrm{M}$ of pterostilbene (solubilized in methanol or encapsulated in nanoemulsion) was added to the apical side while $1.5 \mathrm{~mL}$ of blank HBSS buffer was added to the basolateral side. The experiment was held in an incubator maintained at $37^{\circ} \mathrm{C}$ with $5 \% \mathrm{CO}_{2}$ and $95 \%$ air. Sampling from the basolateral chamber was made at 0, 15, 30, 60, 90, and $120 \mathrm{~min}$. Each time, $750 \mu \mathrm{L}$ of the solution was collected from the basolateral side and replaced with a corresponding volume of fresh HBSS buffer. The trans-epithelial electrical resistance (TEER) value was measured using an EVOM voltohmmeter (Millicell-ERS, Millipore, Bedford, MA, USA) throughout the experiment to ensure the integrity of the monolayer. The integrity of the caco-2 transwell model was indicated when the TEER was larger than $300 \Omega / \mathrm{cm}^{2}$. All the collected samples were immediately extracted with ethyl acetate, dried, and resolubilized with $100 \mu \mathrm{L}$ of methanol before being subject to HPLC analysis. $P_{a p p}$ was calculated based on the following equation:

$$
P_{a p p}=\frac{d Q}{d t} \times \frac{1}{\left(A \times c_{0}\right)}
$$

where $P_{a p p}$ is the apparent permeability coefficient $(\mathrm{cm} / \mathrm{sec}), d Q / d t$ is the linear appearance rate of the compound over $2 \mathrm{~h}(\mu \mathrm{g} / \mathrm{s})$, A is the surface area of the cell monolayer $\left(0.9 \mathrm{~cm}^{2}\right)$, and $c_{0}$ the initial concentration of compound in the donor chamber $(\mu \mathrm{g} / \mathrm{mL})$. 


\subsection{Statistical Analysis}

The data were analyzed using a student's t-test for paired comparisons between the means of samples. $p$ values of $<0.05$ were considered to be significant. The data are shown as the mean \pm standard deviation (SD).

\section{Results and Discussion}

\subsection{Solubility of Pterostilbene in Different Oils}

To maximize the loading concentration of pterostilbene, it is of great importance to realize its solubility in different types of oil, among which the most suitable carrier oil for the development of nanoemulsion could be found. As indicated by the previous investigation, the physical and chemical properties of oil could have a great influence on its ability to solubilize pterostilbene [26]. Specifically, the variation in the structure, length, and saturation degree of their hydrocarbon chain could be critical in determining their ability to solubilize a hydrophobic component [27]. In the present study, seven different oils with various degrees of saturation were selected for the evaluation of being the carrier oil for pterostilbene nanoemulsion (Figure 1). Among the tested oils, medium chain triglycerides (MCT) containing fatty acid with an aliphatic tail of 6-12 carbon and the highest degree of saturation ( $97 \%$ saturated) appeared to be the one that could solubilize the most amount of pterostilbene (208.67 $\pm 3.8 \mathrm{mg} / \mathrm{g}$ oil). According to the result, the ratio of the saturated, monounsaturated, and polyunsaturated fatty acid in the hydrocarbon chain of lipid could be the most influential factor in their ability to dissolve pterostilbene. This phenomenon was again supported when the second-highest solubility of pterostilbene $(164.43 \pm 2.9 \mathrm{mg} / \mathrm{g}$ oil) was found in the coconut oil, which has $86 \%$ saturated fatty acid in its hydrocarbon chain. A similar result was also reported previously where isopropyl myristate, a fully saturated fatty acid esters, had superior capacity to solubilize pterostilbene [26].

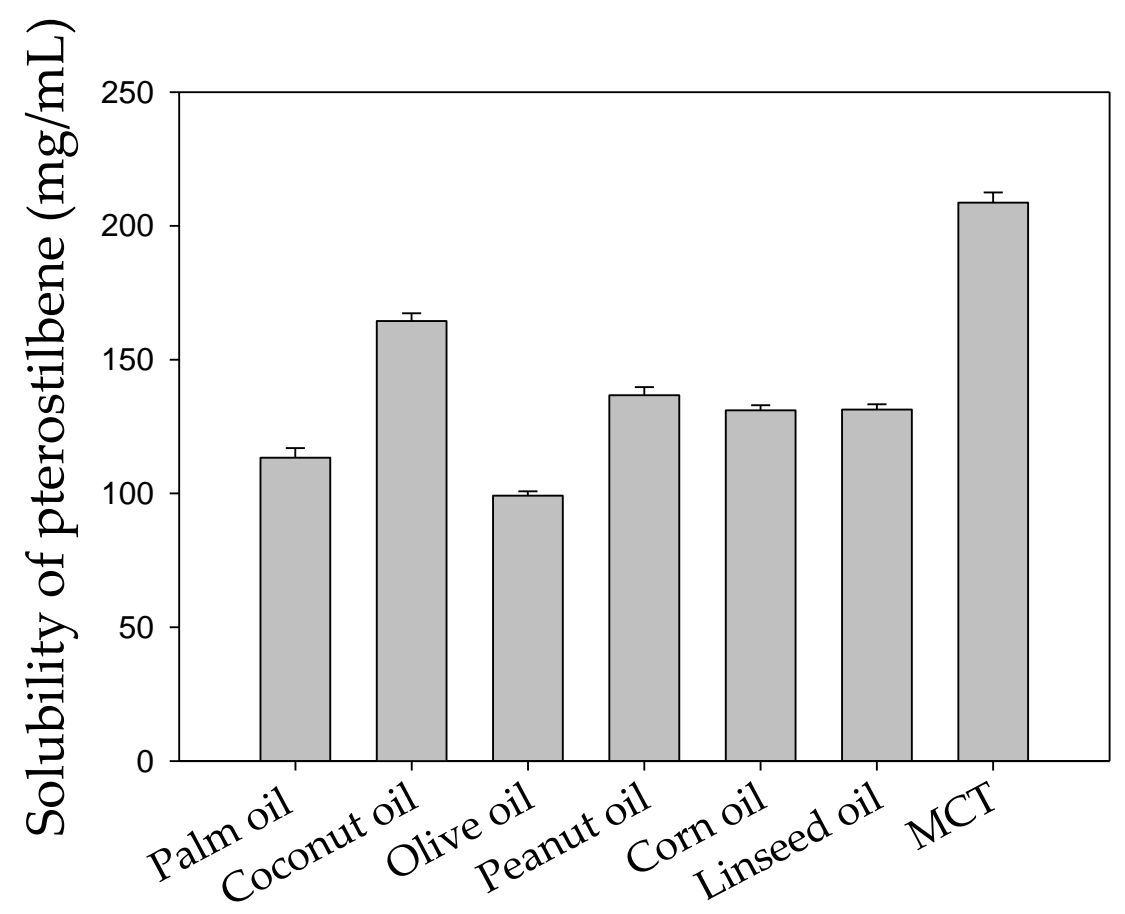

Figure 1. The solubility of pterostilbene in different kinds of oils. Data are presented as mean \pm standard deviation $(n=3)$.

\subsection{Formulation Development from the Pseudoternary Diagram}

MCT being the lipid system that could dissolve the highest amount of pterostilbene was selected as the carrier oil for development of nanoemulsion. A pseudo-ternary phase diagram (Figure A1 in Appendix A) was constructed for the searching of the most cost- 
effective nanoemulsion system to be used to encapsulate pterostilbene. Before mixing with the aqueous phase, the lipid phase was first prepared by mixing the liquid lecithin with MCT at a concentration of $2-10 \%(w / w)$. Based on the result from the diagram, lecithin content lower than $2.5 \%$ would not turn into a stable emulsion system, and thus, at least $3 \%$ of the emulsifier is required for the construction of a stable nanoemulsion system. However, a higher concentration of lecithin in the formulation linearly increased the system viscosity to a point that it became larger than $500 \mathrm{cps}$. and could no longer be further processed by the available high-pressure homogenizer. That is, formulation containing more than $5 \%$ of lecithin was transformed into a semi-liquid gel structure, which could not pass through the narrow channel within the pressurized homogenizer. Keeping lecithin at its minimum requirement $(3 \%)$, stable one-phase emulsion could be formed when the oil phase occupied $30-50 \%$ of the whole system. Since pterostilbene is a hydrophobic compound, a higher ratio of the oil phase in the emulsion formulation could eventually lead to a higher encapsulation rate, and thus, $50 \%$ of the oil phase became the favorable choice for the development of a nanoemulsion system containing pterostilbene.

\subsection{Effect of High-Pressure Homogenization on the Physical Properties and Encapsulation Efficiency of Pterostilbene Nanoemulsion}

To determine the optimum processing parameters, the physical properties including particle size, zeta potential, viscosity, and encapsulation efficiency of pterostilbene in the emulsion were evaluated (Table 1) after being subjected to high-pressure homogenization (HPH). The quality of HPH-produced nanoemulsions was not significantly different after going through 500, 1000, and 1500 bars of pressure (data not shown). Thus, considering energy saving and cost efficiency, the lower processing pressure (500 bar) was selected for the production of pterostilbene nanoemulsion. While the zeta potential and encapsulation efficiency remain unaffected, the droplet size and the system viscosity decreased significantly from $2507.23 \pm 547.31 \mathrm{~nm}$ and $15.45 \pm 0.62 \mathrm{cps}$. to $448.17 \pm 81.51 \mathrm{~nm}$ and $4.2 \pm 0.16$ cps., respectively, after going through 1 cycle of the HPH process. Repeated cycles through the HPH could further reduce the droplet size to approximately $200 \mathrm{~nm}$, which is the generally recognized size for droplets in a nanoemulsion. However, the droplet size reached its minimum at the third cycle, and more processing cycles, instead of decreasing the diameter, caused particle enlargement.

Table 1. The physical characteristic of pterostilbene nanoemulsion with or without high-pressure homogenization $(\mathrm{HPH})$.

\begin{tabular}{|c|c|c|c|c|}
\hline $\begin{array}{c}\text { HPH } \\
\text { (Number of } \\
\text { Cycle) }\end{array}$ & $\begin{array}{l}\text { Particle Size } \\
\quad(\mathrm{nm})\end{array}$ & $\begin{array}{c}\text { Zeta Potential } \\
(\mathrm{mV})\end{array}$ & $\begin{array}{l}\text { Viscosity } \\
\text { (cps) }\end{array}$ & $\begin{array}{c}\text { Encapsulation } \\
\text { Efficiency } \\
(\%)\end{array}$ \\
\hline 0 & $\begin{array}{c}2507.23 \pm 547.31 \\
a\end{array}$ & $-38.52 \pm 0.29 \mathrm{~cd}$ & $15.45 \pm 0.62^{a}$ & $99.08 \pm 1.23^{a}$ \\
\hline 1 & $448.17 \pm 81.51^{b}$ & $-38.19 \pm 0.10^{b}$ & $4.20 \pm 0.16^{b c}$ & $98.19 \pm 1.64^{\mathrm{a}}$ \\
\hline 2 & $366.07 \pm 57.26^{b}$ & $-37.94 \pm 0.15^{\mathrm{a}}$ & $4.30 \pm 0.74 \mathrm{bc}$ & $99.34 \pm 0.85^{\mathrm{a}}$ \\
\hline 3 & $212.63 \pm 13.14^{b}$ & $-38.63 \pm 0.20^{\mathrm{d}}$ & $4.60 \pm 0.17^{b c}$ & $95.45 \pm 2.34^{\mathrm{a}}$ \\
\hline 4 & $272.26 \pm 79.31^{b}$ & $-38.61 \pm 0.17^{\mathrm{d}}$ & $4.00 \pm 0.17^{c}$ & $94.11 \pm 7.75^{\mathrm{a}}$ \\
\hline 5 & $452.30 \pm 35.75^{b}$ & $-38.34 \pm 0.20 b c$ & $5.20 \pm 0.17^{\mathrm{d}}$ & $98.64 \pm 0.95^{\mathrm{a}}$ \\
\hline
\end{tabular}

The same superscripts indicate no significant difference between groups listed in the same row. Different superscripts $(\mathrm{a}, \mathrm{b}, \mathrm{c}$, and d) indicate a significant difference between groups $(p<0.05)$. Data are presented as mean \pm standard deviation $(n=3)$.

This phenomenon could be explained by "The optimal amount theory", which indicated that the smallest droplet size could be reached if the optimum quantity of emulsifier is available to cover the surface area of the droplet [28]. On the contrary, the droplets tend to aggregate with each other to reduce the surface area if the amount of emulsifier is not enough to cover them. In the present study, $3 \%$ of the lecithin was used to stabilize an emulsion system with $50 \%$ oil phase (high oil content). As the $\mathrm{HPH}$ process is to mechanically 
break down the emulsion droplet, it also increased the surface area needed to be coated by an emulsifier. Thus, the result obtained from the experiment indicated that 3 cycles of HPH could produce droplets with a total surface area that matches the surface coverage capacity of the amount of lecithin added to the formulation. A similar result was also found by Yan et al. where lecithin could significantly decrease the emulsion droplet size at lower but not higher processing pressure [29].

The significant change in the viscosity of emulsion after HPH could be attributed to the droplet size reduction, which allows higher mobility in the bulk phase. The viscosity appeared to be highly sensitive to the major particle size reduction during its first pass through HPH resulting in a great decrease of viscosity. However, a further decrease of the particle size in subsequent cycles showed a less significant viscosity variation indicating that its viscosity decrease in an exponential trend with the decreasing particle size. Moreover, the reduction of emulsion viscosity caused by the HPH may not only be related to the change of its droplet size, but also to the flow properties of lecithin molecules. $\mathrm{HPH}$ processing provides sufficient energy to disrupt covalent bonds and non-covalent interactive forces, which could disrupt the aggregation of the lecithin molecule and its network structures. Thus, the mobility of lecithin molecules increased with the increased cycles of HPH processing.

The fact that zeta potential and encapsulation efficiency was not significantly changed by the HPH treatment indicated that these properties were dependent on other influential factors such as the charge property of the emulsifier and solubility of pterostilbene in the carrier oil, respectively. That is, the zeta potential and the capacity of nanoemulsion to encapsulate pterostilbene remained stable over the HPH since neither of these factors could be easily altered by HPH.

\subsection{The Stability Study}

The stability of pterostilbene was evaluated after 3 passes through the HPH. A few important stability indicators including droplet size, zeta potential, viscosity, and compound encapsulation efficiency were measured over a period of 28 days of storage (Figure 2). The size of an emulsion droplet is a commonly studied property to examine the ability of a system to cope with instability mechanisms, such as Ostwald ripening, coalescence, and flocculation, which leads to phase separation [30]. According to the theory of Stoke's law, the stability of an emulsion system is inversely related to the size of its droplets. In the present study, $\mathrm{HPH}$ significantly reduced the droplet size of pterostilbene emulsion and, thus, should effectively increase its stability. Over 28 days of storage, the droplet size along with the viscosity of the coarse emulsion (non HPH treated) continuously increased indicating the propagation of certain destabilization mechanisms. On the other hand, pterostilbene nanoemulsion produced using HPH exhibited good stability with no significant change in the average droplet diameter as well as its systemic viscosity.

A similar phenomenon was also observed in the testing of encapsulation efficiency where the HPH-processed nanoemulsion maintained its high rate of pterostilbene encapsulation ability, while more than $10 \%$ of the pterostilbene was lost from the coarse emulsion (Figure 2D). Zeta potential, representing the charge density on the droplet surface, however, is directly affected by the charge property of the molecule covering the droplet surface. As the structure of lecithin, a negatively charged emulsifier, was not affected by the HPH process, the droplet surface covered by such molecules then remained constant regardless of the high-pressure treatment. 


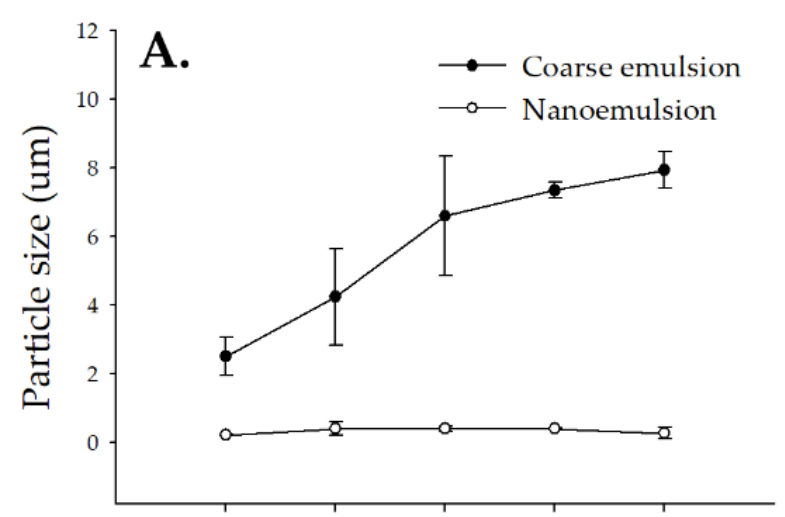

day 0 day 7 day 14 day 21 day 28

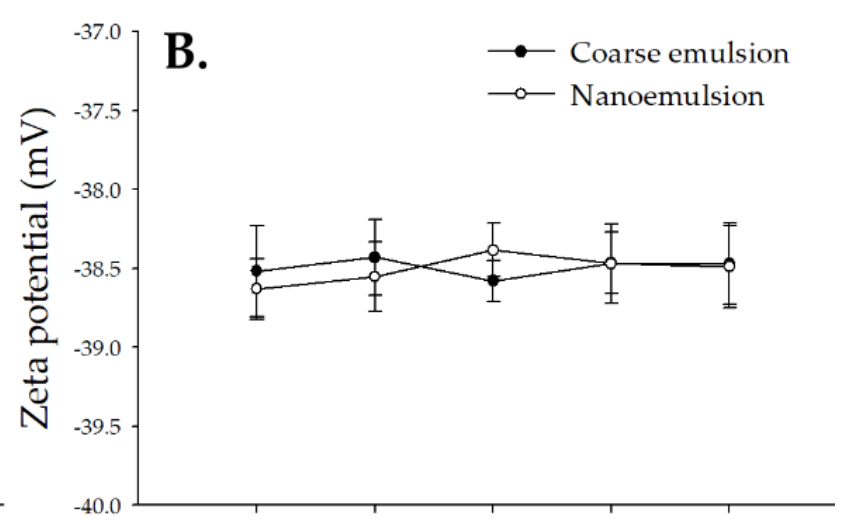

day 0 day 7 day 14 day 21 day 28

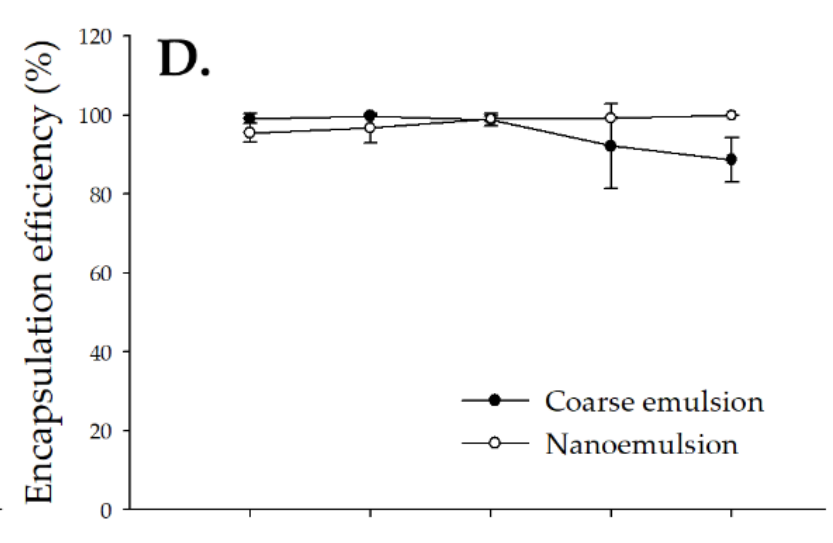

day 0 day 7 day 14 day 21 day 28

Figure 2. The stability of the (A) particle size, (B) zeta potential, (C) viscosity, and (D) encapsulation efficiency of pterostibene nanoemulsion over 28 days of storage.

\subsection{Effect of Nanoemulsion on the Antioxidant Capacity of Pterostilbene}

Pterostilbene was reported to exert many of its therapeutic effects through its intrinsic antioxidant ability [31-34]. However, pieces of literature also suggested that the bioactive ingredients with strong antioxidant activity are prone to various oxidation stress when exposed to the complicated combination of molecular status and environmental conditions [35]. To improve the stability of the bioactive component, various delivery systems, such as emulsion, nanoparticles, microcapsules, etc., were developed by researchers to enhance the oxidative stability of target compounds [36]. With good storage stability, nanoemulsion that contained more than $9 \%$ of pterostilbene was further subjected to an evaluation of its antioxidant capacity using DPPH radical scavenging assay. According to the result, pterostilbene in nanoemulsion showed significantly higher antioxidant activity at a concentration in the range of $0.5-3 \mathrm{mg} / \mathrm{mL}$ than its corresponding free compound in an aqueous environment (Figure 3). Through offering better protection against various oxidative stress, the nanoemulsion system showed superior ability to enhance the antioxidant activity of pterostilbene even at a low compound concentration, which could indicate an expected improvement in the therapeutic efficiency if used for disease treatment. 


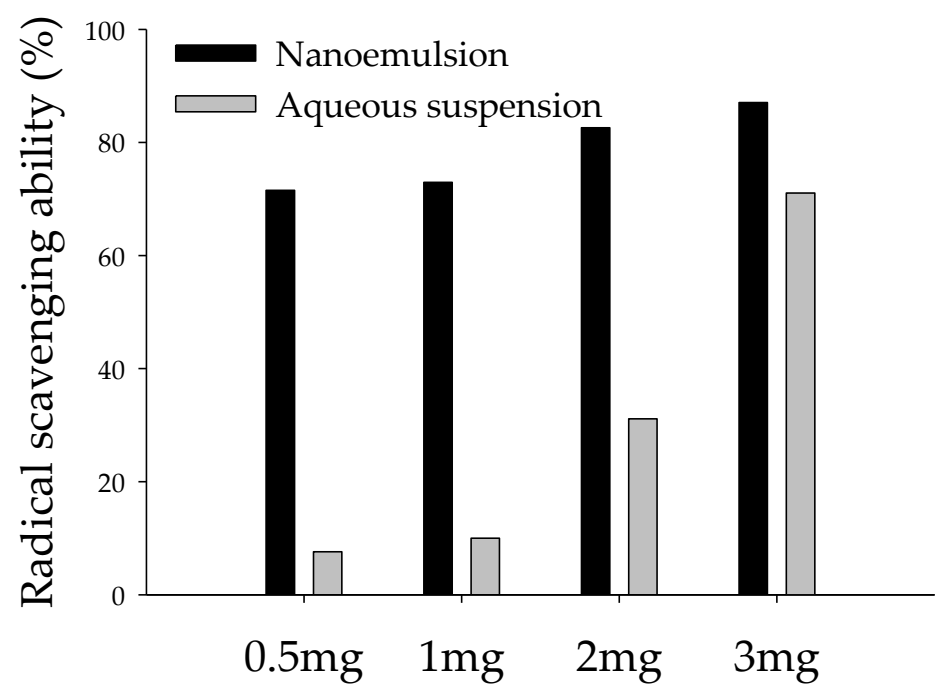

Figure 3. Antioxidant capacity of pterostilbene nanoemulsion and water suspension.

\subsection{Effect of Nanoemulsion on the In Vitro Bioaccessibility of Pterostilbene}

The oral bioavailability of a bioactive component is positively related to the fraction of its ingested dosage that becomes accessible for intestinal absorption [8]. The bioaccessibility, however, is conclusively affected by many physiochemical factors. For most bioactive compound, its solubility, stability, and retention rate in the gastric environment largely influences its probability to become accessible for intestinal absorption. Pterostilbene being a hydrophobic compound is poorly soluble in the aqueous environment, and thus, its oral bioavailability is rather unsatisfactory for most of the therapeutic applications $[37,38]$. Due to their higher solubility in lipids, the bioaccessibility and oral bioavailability of hydrophobic components are usually improved with the presence of lipid during digestion. Therefore, many of the lipid-based oral formulations were developed to encapsulate compound with poor aqueous solubility. In this sense, in vitro lipolysis study then become a commonly used digestion model that allow rapid evaluation of oral formulations in terms of their effects on the bioaccessibility of a bioactive component.

Accompanying the lipid digestion, the $\mathrm{pH}$ value continuously decreased due to the release of fatty acid from the lipid hydrolysis. To keep the optimum working $\mathrm{pH}$ for the enzymatic activity, $\mathrm{NaOH}$ was repeatedly added to the digestion buffer, and thus, the digestion kinetic could be revealed by plotting the added volume of $\mathrm{NaOH}$ against time. In this study, the digestion of lipid in the pterostilbene nanoemulsion proceeded at a much faster pace than its suspension in the bulk oil and water (Figure 4A). This result was found to be similar to the previously reported work where nanoemulsion provided a much larger surface area for the lipase absorption than the bulk oil and, thus, leads to higher digestion rate [8]. As lipids are hydrolyzed by lipase, the produced fatty acids along with bile salts are then transformed into the micelle structure, which is the main component absorbed by enterocytes. During the micelle formation, the nearby hydrophobic compound can also be incorporated into the hydrophobic micelle core and then collectively becomes available for intestinal uptake. Here, approximately $84.94 \%$ of the pterostilbene in the nanoemulsion becomes bioaccessible whereas in the bulk oil and water suspension only $19.44 \%$ and $8.46 \%$ of it is available for intestinal absorption (Figure 4B). Results from this part of the work strongly suggested that the faster lipid digestion rate could also result in the significantly higher pterostilbene bioaccessibility. 

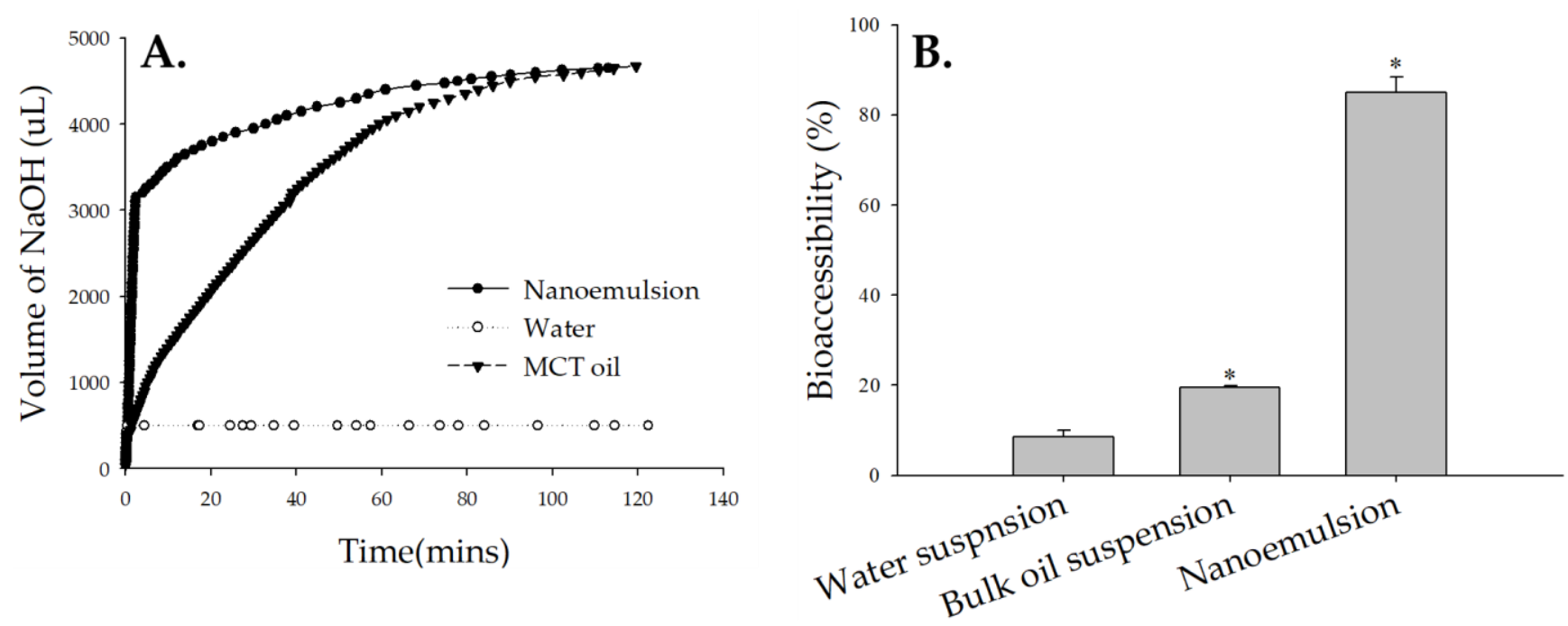

Figure 4. In vitro lipolysis profiles of pterostilbene nanoemulsion, bulk oil, and water suspension. (A) The digestion kinetics expressed as the amount of added $\mathrm{NaOH}$ as a function of time. (B) Percent pterostilbene bioaccessibility relative to the original dose in the nanoemulsion, bulk oil, and water. Data are presented as mean \pm standard deviation $(n=3) .{ }^{*}=p<0.01$.

\subsection{Effect of Nanoemulsion on the Transport of Pterostilbene through the Caco-2 Monolayer}

The cytotoxicity effect of pterostilbene on the caco-2 cell examined using MTT assay can be seen in Figure 5A. MTT is a yellow tetrazolium salt that is oxidized by mitochondria and transformed into a purple formazan product, which could be quantified spec-metrically. As damaged or dead cells show reduced or no dehydrogenase activity, the cell viability decrease of the absorbance intensity from the healthy cells could be an indicator of cellular toxicity. In general, cells with an intensity decrease of less than $20 \%$ were regarded as non-toxic to the cells whereas more than $50 \%$ were thought to be cytotoxic causing the reduction in mitochondrial activity [39]. As shown in Figure 5A, there was a decrease in cell viability with the increasing pterostilbene concentration from 25 to $200 \mu \mathrm{M}$. More than $80 \%$ of cells remained viable at a concentration less than $50 \mu \mathrm{M}$ indicating a good tolerance of caco-2 cells at this level. Therefore, pterostilbene of $50 \mu \mathrm{M}$ was selected as the working concentration for the subsequent caco-2 monolayer study to avoid the overestimation of the transport rate due to cell damage.

To properly carry out the transport study using caco-2 monolayer assay, it is important to ensure the membrane integrity over the cultivation period, during which cells slowly differentiate themselves into a structure similar to the intestine epithelium. Thus, a well-differentiated monolayer structure should have a tight junction formed between the adjacent cells and could be monitored by measuring its trans-epithelial electrical resistance (TEER) value. To ensure the membrane integrity, monolayer with a TEER value higher than $350 \Omega / \mathrm{cm}^{2}$ is used for the transport study. As shown in Figure 5B, the TEER value of the caco- 2 cells continues to increase over the 21 days of the cultivation period. At the time of the transport experiment, all monolayer had a TEER value around $600 \Omega / \mathrm{cm}^{2}$ indicating there should be no excess compound permeability caused by a defect of the cell lining.

The apparent permeability $\left(P_{a p p}\right)$ of most compounds through the intestinal lining falls within the range of $1 \times 10^{-7}$ to $1 \times 10^{-5} \mathrm{~cm} / \mathrm{s}$. Compounds exhibiting a $P_{a p p}$ value at the range of $1 \times 10^{-7} \mathrm{~cm} / \mathrm{s}$ are considered poorly transported, whereas those well-transported ones had a $P_{a p p}$ value higher than $1 \times 10^{-5} \mathrm{~cm} / \mathrm{s}$ [40]. As shown in Figure 5C, the $P_{\text {app }}$ values for the tested formulations fall within the range of a high transport range. Usually, compound solubilized in an organic solvent (in this case methanol) is expected to exhibit the highest apparent permeability since the higher solubility in the solvent contributes to a reduction in the resistance of the mass transfer across the cell monolayer [41]. Here, the apparent permeability of pterostilbene encapsulated in the nanoemulsion system closely approximated the methanol-solubilized pterostilbene. This result indicated that the nanoemulsion system, 
without using toxic organic solvent, had successfully improved the transport of pterostilbene across the intestinal lining since the transport of the unencapsulated pterostilbene in the aqueous system was unmeasurable (beyond the instrumental detection level).
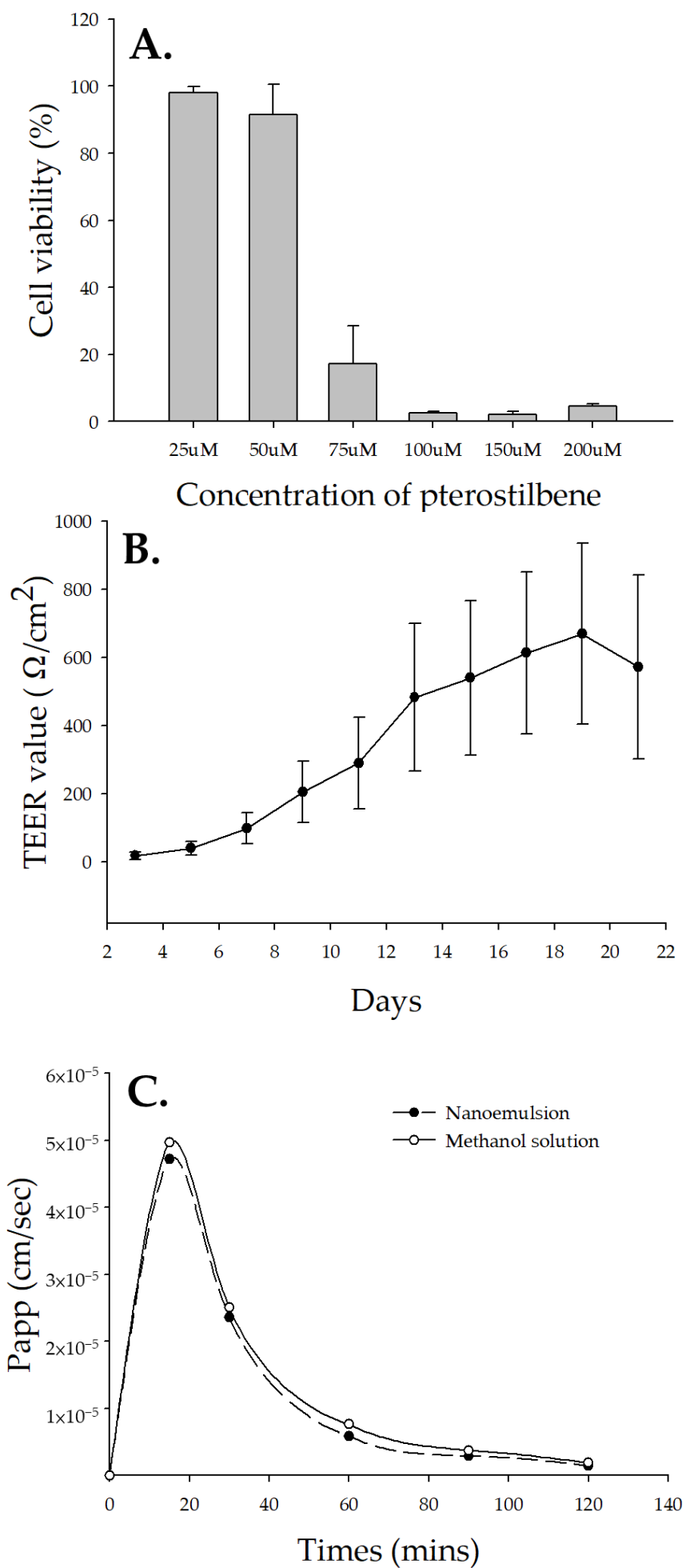

Figure 5. (A) Cell viability after being treated with $25-200 \mu \mathrm{M}$ of pterostilbene. (B) The transepithelial electrical resistance (TEER) value of caco-2 monolayer over 22 days of cultivation period and $(\mathbf{C})$ transport kinetics of pterostilbene through the caco-2 monolayer. 


\section{Conclusions}

Nanoemulsion with a high loading of pterostilbene was successfully developed through optimization in the formulation and processing parameters. The result from this work has also proven that the stability, bioaccessibility, and intestinal transport rate of pterostilbene could be significantly improved when encapsulated in the nanoemulsion system. These results have important implications in the design of lipid-based encapsulation for pterostilbene in food-related applications. It is confirmed that the encapsulation of hydrophobic compounds in the emulsion-based delivery system is an effective means to increase their solubility and bioavailability and to protect them from degradation.

Author Contributions: Conception and design: Q.H. and Y.T.; financial support: Y.T.; writing and censorship: F.-M.S. and Y.-J.C.; performed research and analyzed data: F.-M.S.; manuscript editing: J.-Y.H. All authors have read and agreed to the published version of the manuscript.

Funding: This work is supported by the Taiwan Ministry of Science and Technology (Grant No. 105-2320-B-002-004-).

Institutional Review Board Statement: Not applicable.

Informed Consent Statement: Not applicable.

Data Availability Statement: All data used to support the findings of this study are included within the article.

Conflicts of Interest: The authors declare no conflict of interest.

\section{Appendix A}

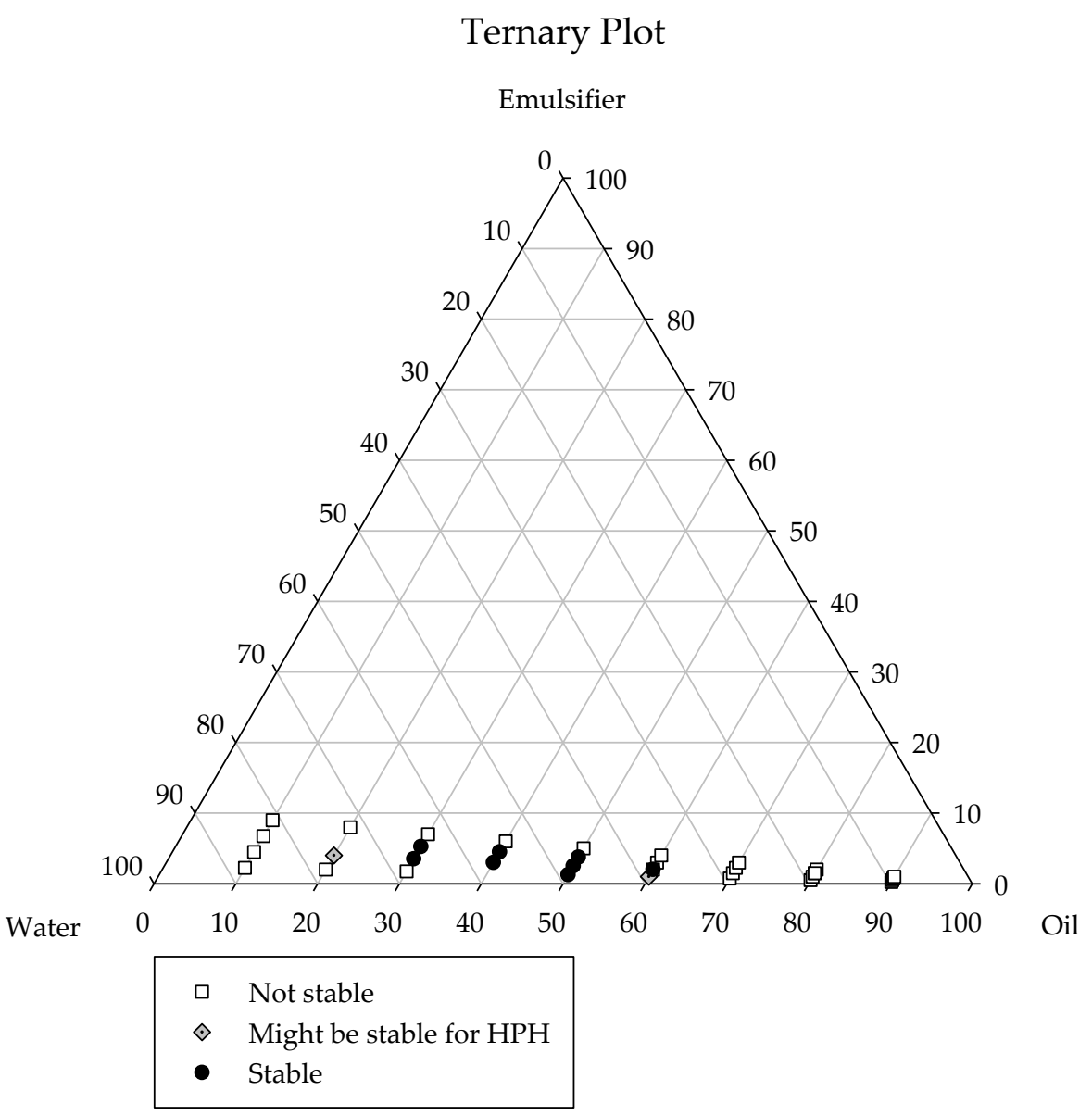

Figure A1. The ternary phase diagram of coarse emulsion. 


\section{References}

1. Adrian, M.; Jeandet, P.; Douillet-Breuil, A.-C.; Tesson, L.; Bessis, R. Stilbene content of mature Vitis vinifera berries in response to UV-C elicitation. J. Agric. Food Chem. 2000, 48, 6103-6105. [CrossRef] [PubMed]

2. Manickam, M.; Ramanathan, M.; Jahromi, M.; Chansouria, J.P.N.; Ray, A.B. Antihyperglycemic Activity of Phenolics fromPterocarpus marsupium. J. Nat. Prod. 1997, 60, 609-610. [CrossRef] [PubMed]

3. Rimando, A.M.; Kalt, W.; Magee, J.B.; Dewey, J.; Ballington, J.R. Resveratrol, pterostilbene, and piceatannol in vaccinium berries. J. Agric. Food Chem. 2004, 52, 4713-4719. [CrossRef] [PubMed]

4. McCormack, D.; McFadden, D. A Review of Pterostilbene Antioxidant Activity and Disease Modification. Oxidative Med. Cell. Longev. 2013, 2013, 1-15. [CrossRef] [PubMed]

5. Chang, J.; Rimando, A.; Pallas, M.; Camins, A.; Porquet, D.; Reeves, J.; Casadesus, G. Low-dose pterostilbene, but not resveratrol, is a potent neuromodulator in aging and Alzheimer's disease. Neurobiol. Aging 2012, 33, 2062-2071. [CrossRef]

6. Chiou, Y.-S.; Tsai, M.-L.; Nagabhushanam, K.; Wang, Y.-J.; Wu, C.-H.; Ho, C.-T.; Pan, M.-H. Pterostilbene is More Potent than Resveratrol in Preventing Azoxymethane (AOM)-Induced Colon Tumorigenesis via Activation of the NF-E2-Related Factor 2 (Nrf2)-Mediated Antioxidant Signaling Pathway. J. Agric. Food Chem. 2011, 59, 2725-2733. [CrossRef]

7. Choo, Q.-Y.; Yeo, S.C.M.; Ho, P.C.; Tanaka, Y.; Lin, H.-S. Pterostilbene surpassed resveratrol for anti-inflammatory application: Potency consideration and pharmacokinetics perspective. J. Funct. Foods 2014, 11, 352-362. [CrossRef]

8. Ting, Y.; Zhao, Q.; Xia, C.; Huang, Q. Using in Vitro and in Vivo Models to Evaluate the Oral Bioavailability of Nutraceuticals. J. Agric. Food Chem. 2015, 63, 1332-1338. [CrossRef]

9. Shah, P.; Bhalodia, D.; Shelat, P. Nanoemulsion: A pharmaceutical review. Syst. Rev. Pharm. 2010, 1, 24. [CrossRef]

10. Aboofazeli, R. Nanometric-Scaled Emulsions (Nanoemulsions). Iran. J. Pharm. Res. 2010, 9, 325-326.

11. Humphries, J.; Pizzi, D.; Sonderegger, S.E.; Fletcher, N.L.; Houston, Z.H.; Bell, C.A.; Thurecht, K.J. Hyperbranched poly (2oxazoline) s and poly (ethylene glycol): A structure-Activity comparison of biodistribution. Biomacromolecules 2020, 21, 3318-3331. [CrossRef] [PubMed]

12. Lin, W.; Kampf, N.; Goldberg, R.; Driver, M.J.; Klein, J. Poly-phosphocholinated liposomes form stable superlubrication vectors. Langmuir 2019, 35, 6048-6054. [CrossRef] [PubMed]

13. Noureddine, A.; Maestas-Olguin, A.; Saada, E.A.; LaBauve, A.E.; Agola, J.O.; Baty, K.E.; Doudna, J.A. Engineering of monosized lipid-coated mesoporous silica nanoparticles for CRISPR delivery. Acta Biomater. 2020, 114, 358-368. [CrossRef] [PubMed]

14. Letona, C.A.M.; Luo, K.; Jeong, K.-B.; Adra, H.J.; Park, C.-S.; Kim, Y.-R. Effect of lecithin on the spontaneous crystallization of enzymatically synthesized short-chain amylose molecules into spherical microparticles. Polymers 2019, 11, 264. [CrossRef] [PubMed]

15. Ting, Y.; Li, C.C.; Pan, M.-H.; Ho, C.-T.; Huang, Q. Effect of a Labile Methyl Donor on the Transformation of 5-Demethyltangeretin and the Related Implication on Bioactivity. J. Agric. Food Chem. 2013, 61, 8090-8097. [CrossRef]

16. Hoeller, S.; Sperger, A.; Valenta, C. Lecithin based nanoemulsions: A comparative study of the influence of non-ionic surfactants and the cationic phytosphingosine on physicochemical behaviour and skin permeation. Int. J. Pharm. 2009, 370, 181-186. [CrossRef]

17. Klang, V.; Valenta, C. Lecithin-based nanoemulsions. J. Drug Deliv. Sci. Technol. 2011, 21, 55-76. [CrossRef]

18. Zhou, H.; Yue, Y.; Liu, G.; Li, Y.; Zhang, J.; Gong, Q.; Yan, Z.; Duan, M. Preparation and Characterization of a Lecithin Nanoemulsion as a Topical Delivery System. Nanoscale Res. Lett. 2009, 5, 224-230. [CrossRef]

19. Donsì, F.; Sessa, M.; Mediouni, H.; Mgaidi, A.; Ferrari, G. Encapsulation of bioactive compounds in nanoemulsion-based delivery systems. Procedia Food Sci. 2011, 1, 1666-1671. [CrossRef]

20. Liu, B. Identification of Oxidative Products of Pterostilbene and 3'-Hydroxypterostilbene In Vitro and Evaluation of AntiInflammatory and Anti-Cancer Cell Proliferative Activity. Master's Thesis, Rutgers University-Graduate School, New Brunswick, NJ, Canada, 2014.

21. Diane, J.M.M.; Burgess, J. Vitamin E nanoemulsions characterization and analysis. Int. J. Pharm. 2014, 465, 455-463. [CrossRef]

22. Ahmed, K.; Li, Y.; McClements, D.J.; Xiao, H. Nanoemulsion- and emulsion-based delivery systems for curcumin: Encapsulation and release properties. Food Chem. 2012, 132, 799-807. [CrossRef]

23. Sharma, S.; Sahni, J.K.; Ali, J.; Baboota, S. Effect of high-pressure homogenization on formulation of TPGS loaded nanoemulsion of rutin-Pharmacodynamic and antioxidant studies. Drug Deliv. 2015, 22, 541-551. [CrossRef] [PubMed]

24. Silva, H.D.; Poejo, J.; Pinheiro, A.C.; Donsì, F.; Serra, A.T.; Duarte, C.M.; Ferrari, G.; Cerqueira, M.A.; Vicente, A.A. Evaluating the behaviour of curcumin nanoemulsions and multilayer nanoemulsions during dynamic in vitro digestion. J. Funct. Foods 2018, 48, 605-613. [CrossRef]

25. Hubatsch, I.; E Ragnarsson, E.G.; Artursson, P. Determination of drug permeability and prediction of drug absorption in Caco-2 monolayers. Nat. Protoc. 2007, 2, 2111-2119. [CrossRef]

26. Zhang, Y.; Shang, Z.; Gao, C.; Du, M.; Xu, S.; Song, H.; Liu, T. Nanoemulsion for Solubilization, Stabilization, and In Vitro Release of Pterostilbene for Oral Delivery. AAPS PharmSciTech 2014, 15, 1000-1008. [CrossRef]

27. Bali, V.; Ali, M.; Ali, J. Study of surfactant combinations and development of a novel nanoemulsion for minimising variations in bioavailability of ezetimibe. Colloids Surfaces B Biointerfaces 2010, 76, 410-420. [CrossRef] 
28. Shi, A.-M.; Li, D.; Wang, L.-J.; Li, B.-Z.; Adhikari, B. Preparation of starch-based nanoparticles through high-pressure homogenization and miniemulsion cross-linking: Influence of various process parameters on particle size and stability. Carbohydr. Polym. 2011, 83, 1604-1610. [CrossRef]

29. Yan, B.; Park, S.H.; Balasubramaniam, V.M. Influence of high pressure homogenization with and without lecithin on particle size and physicochemical properties of whey protein-based emulsions. J. Food Process. Eng. 2017, 40, e12578. [CrossRef]

30. Chebil, A.; Desbrières, J.; Nouvel, C.; Six, J.-L.; Durand, A. Ostwald ripening of nanoemulsions stopped by combined interfacial adsorptions of molecular and macromolecular nonionic stabilizers. Coll. Surfaces A Physicochem. Eng. Aspects 2013, 425, 24-30. [CrossRef]

31. Benlloch, M.; Obrador, E.; Valles, S.L.; Rodriguez, M.L.; Sirerol, J.A.; Alcácer, J.; Pellicer, J.A.; Salvador, R.; Cerdá, C.; Sáez, G.T.; et al. Pterostilbene Decreases the Antioxidant Defenses of Aggressive Cancer Cells In Vivo: A Physiological Glucocorticoids- and Nrf2-Dependent Mechanism. Antioxid. Redox Signal. 2016, 24, 974-990. [CrossRef]

32. Elango, B.; Dornadula, S.; Paulmurugan, R.; Ramkumar, K.M. Pterostilbene Ameliorates Streptozotocin-Induced Diabetes through Enhancing Antioxidant Signaling Pathways Mediated by Nrf2. Chem. Res. Toxicol. 2016, 29, 47-57. [CrossRef] [PubMed]

33. Li, J.; Deng, R.; Hua, X.; Zhang, L.; Lu, F.; Coursey, T.G.; Pflugfelder, S.C.; Li, D.-Q. Blueberry Component Pterostilbene Protects Corneal Epithelial Cells from Inflammation via Anti-oxidative Pathway. Sci. Rep. 2016, 6, 19408. [CrossRef] [PubMed]

34. Xue, E.-X.; Lin, J.-P.; Zhang, Y.; Sheng, S.-R.; Liu, H.-X.; Zhou, Y.-L.; Xu, H. Pterostilbene inhibits inflammation and ROS production in chondrocytes by activating Nrf2 pathway. Oncotarget 2017, 8, 41988-42000. [CrossRef] [PubMed]

35. McClements, D.J. Advances in nanoparticle and microparticle delivery systems for increasing the dispersibility, stability, and bioactivity of phytochemicals. Biotechnol. Adv. 2020, 38, 107287. [CrossRef] [PubMed]

36. Martínez-Ballesta, M.; Gil-Izquierdo, A.; García-Viguera, C.; Domínguez-Perles, R. Nanoparticles and Controlled Delivery for Bioactive Compounds: Outlining Challenges for New "Smart-Foods" for Health. Foods 2018, 7, 72. [CrossRef] [PubMed]

37. Bethune, S.J.; Schultheiss, N.; Henck, J.-O. Improving the Poor Aqueous Solubility of Nutraceutical Compound Pterostilbene through Cocrystal Formation. Cryst. Growth Des. 2011, 11, 2817-2823. [CrossRef]

38. Peng, R.-M.; Lin, G.-R.; Ting, Y.; Hu, J.-Y. Oral delivery system enhanced the bioavailability of stilbenes: Resveratrol and pterostilbene. BioFactors 2018, 44, 5-15. [CrossRef]

39. Wahlang, B.; Pawar, Y.B.; Bansal, A.K. Identification of permeability-related hurdles in oral delivery of curcumin using the Caco-2 cell model. Eur. J. Pharm. Biopharm. 2011, 77, 275-282. [CrossRef]

40. Jia, L.; Wong, H. In vitro and in vivo assessment of cellular permeability and pharmacodynamics of S-nitrosylated Captopril, a nitric oxide donor. Br. J. Pharmacol. 2001, 134, 1697-1704. [CrossRef]

41. Sessa, M.; Balestrieri, M.L.; Ferrari, G.; Servillo, L.; Castaldo, D.; D'Onofrio, N.; Tsao, R. Bioavailability of encapsulated resveratrol into nanoemulsion-based delivery systems. Food Chem. 2014, 147, 42-50. [CrossRef] 